\title{
Limitación de la apertura bucal por hiperplasia de apófisis coronoides: reporte de un caso
}

\author{
Limitation of oral opening related to coronoid process \\ hyperplasia: a case report
}

Recibido: 2018/01/10. Aceptado: 2018/02/28. Publicado: 2018/03/15

\section{Fernando Sandoval Portilla ${ }^{1}$ \\ Christopher Naranjo Cajamarca ${ }^{2}$ Fernando Sandoval Vernimmen ${ }^{3}$}

1 Universidad San Francisco de Quito, Colegio de Ciencias de la Salud, Escuela de Odontología, Clínica Odontológica, Campus Cumbayá, oficina CO 106, casilla postal 17-1200-841. Quito-Ecuador.

Correo electrónico: fjose.sandoval@gmail.com

2 Universidad San Francisco de Quito, Colegio de Ciencias de la Salud, Escuela de Odontología, Clínica Odontológica, Campus Cumbayá, oficina CO 106, casilla postal 17-1200-841. Quito-Ecuador.

Correo electrónico: chrisnaranjo_1@yahoo.com

3 Universidad San Francisco de Quito, Colegio de Ciencias de la Salud, Escuela de Odontología, Clínica Odontológica, Campus Cumbayá, oficina CO 106, casilla postal 17-1200-841. Quito-Ecuador.

Correo electrónico: achavez@usfq.edu.ec 


\section{Resumen}

La hiperplasia de los procesos coronoideos es una afección muy rara, caracterizada por la elongación de las coronoides en donde se produce un contacto prematuro con la superficie posterior del hueso cigomático. Esto dará como resultado la restricción mecánica de la apertura bucal. El tratamiento de elección es la coronoidectomia intraoral de forma bilateral. En este artículo reportamos el caso de un paciente de 15 años con una marcada limitación de la apertura bucal, donde se le realizó resección bilateral de los procesos coronoideos de forma intra oral.

Palabras claves: hiperplasia de proceso coronoideo, coronoidectomia, coronoidotomia, apertura bucal

\section{Abstract}

The hyperplasia of the coronoid process is a very rare condition, characterized by an elongated coronoid process, where the contact of the coronoid process with the posterior surface of the zygomatic bone will result in a mechanical restriction of the mouth opening. The treatment of choice is bilateral coronoidectomy.

In this article, we report the case of a 15-year-old patient with a marked limitation of the oral opening, where bilateral resection of the coronoid process was performed intraoral.

Keywords: coronoid process hyperplasia, coronoidectomy, coronoidotomy, buccal opening 


\section{Introducción}

La hiperplasia de apófisis coronoides es una anomalía poco frecuente que causa la reducción progresiva de la apertura bucal. Se define como un alargamiento anormal de los procesos coronoides mandibulares con características histológicas óseas normales, esta puede ser unilateral o bilateral $\mathbf{1 , 2}^{2}$.

La principal característica clínica es la limitación progresiva de la apertura bucal debido a la interferencia entre la apófisis coronoides alargada y la superficie temporal del hueso cigomatico y/o la superficie interna del arco cigomático 1,2,3.

Esta entidad fue descrita por primera vez por Von Langen-beck en 1853, y publicada en 1963 por Rowe, la describe como una anormalidad de los procesos coronoideos, dividiéndolos en 2 tipos, los causados por el desarrollo anormal óseo y debido a cambios neoplásicos, estos últimos se caracterizan por ser unilaterales (enfermedad de Jacob) mientras que los causados por el desarrollo óseo son de forma bilateral $\mathbf{1 , 2 , 3 , 4}$.

Aproximadamente el $80 \%$ de los pacientes afectados por hiperplasia de coronoides son hombres, la limitación progresiva de la apertura bucal generalmente comienza a notarse en la pubertad. 5,6. La etiología de hiperplasia de coronoides no está del todo determinada, se han propuesto algunas teorías como el aumento de la actividad muscular del temporal, trastornos de la articulación temporomandibular u otras enfermedades relacionadas a la articulación temporomandibular, así como también el estímulo endocrino que se inicia en la pubertad puede ser de importancia para esta condición, el trauma facial se ha propuesto como uno de los factores para hiperplasia de coronoides por la limitación del desplazamiento mandibular, aunque esto parece tener menor importancia. ${ }^{\mathbf{5 , 6} \text {. }}$

El Tratamiento de la hiperplasia de los procesos coronoideos es principalmente quirúrgico mediante coronoidectomia bilateral por vía intraoral mediante resección de las apófisis coronoides. ${ }^{6,7}$.

El objetivo de este artículo fue describir la técnica de resección de apófisis coronoides de forma intra oral, logrando excelentes resultados tanto estéticos y funciónales a largo plazo.

\section{Caso clínico}

Paciente masculino de 15 años de edad acudió a la consulta del servicio de cirugía maxilofacial donde la madre manifestó que el niño tiene una marcada limitación de la apertura bucal, la misma que ha ido progresando en este último año. Paciente asintomático, pero manifestó que presenta problemas para alimentarse y realizar una adecuada higiene oral. Madre refirió que anteriormente acudieron a diferentes especialistas, intentando mejorar la apertura bucal mediante terapia física sin tener ningún resultado, inclusive se le diagnosticó como anquilosis de la articulación temporomandibular

Características clínicas

Limitación del movimiento mandibular que no es absoluto.

La limitación aumenta progresivamente durante un período de años.

Afecta más al sexo masculino.

El trastorno es asintomático.

El inicio de la limitación se produce alrededor de la pubertad.

No hay antecedente de trauma.

La excursión lateral esta extremadamente limitada o ausente.

La articulación temporomandibular se presenta normal al examen radiográfico. 
en donde se le propuso condilectomia para mejorar la apertura, por lo que decidieron acudir a la consulta de nuestro servicio para una segunda opinión.

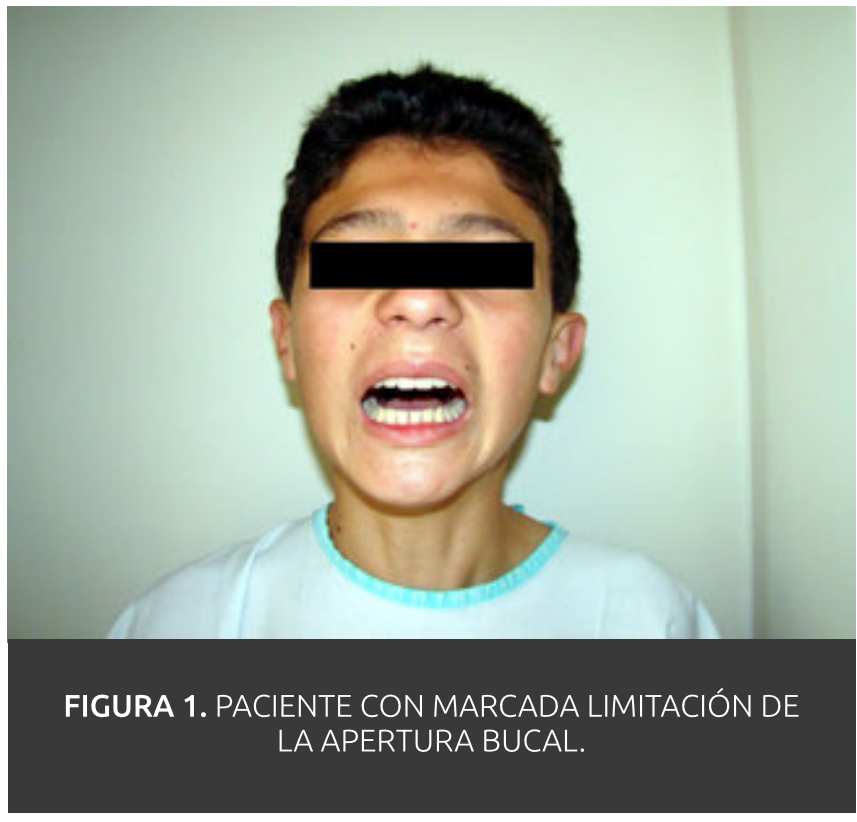

Al examen físico el paciente presentó una apertura bucal de $15 \mathrm{~mm}$ que al forzarla no se logró ninguna variación (figura 1).

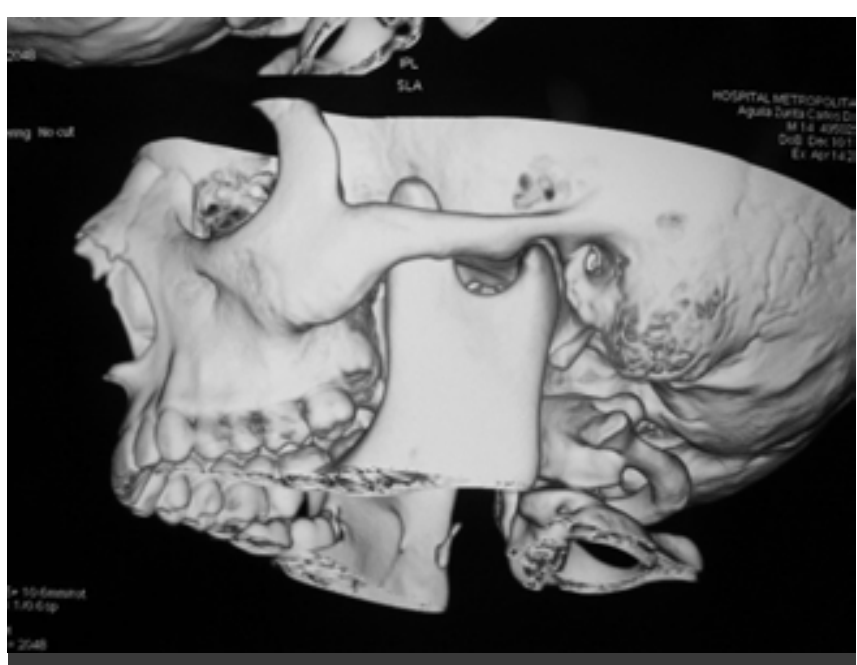

FIGURA 2. T.C SE EVIDENCIA GRAN ELONGACIÓN DEL PROCESO CORONOIDEO.

Al estudio radiográfico y por imágenes (TC) se evidencia una elongación considerable de los procesos coronoideos que sobrepasan el nivel del arco cigomático (figura 2).
Se llega al diagnóstico de hiperplasia de los procesos coronoideos bilaterales y se propone coronoidectomía bilateral, mediante abordaje intraoral bajo anestesia general con intubación naso traqueal con utilización de fibroscopio por la muy marcada limitación de la apertura bucal, en los casos en que una laringoscopias sea difícil, peligrosa o no se la pueda realizar la utilización de fibroscopio es de vital importancia, ya que nos permite realizar una técnica cómoda, a traumática y con visión directa de las estructuras laríngeas, reduciendo así las complicaciones inherentes a una técnica de intubación nasotraqueal a ciegas.

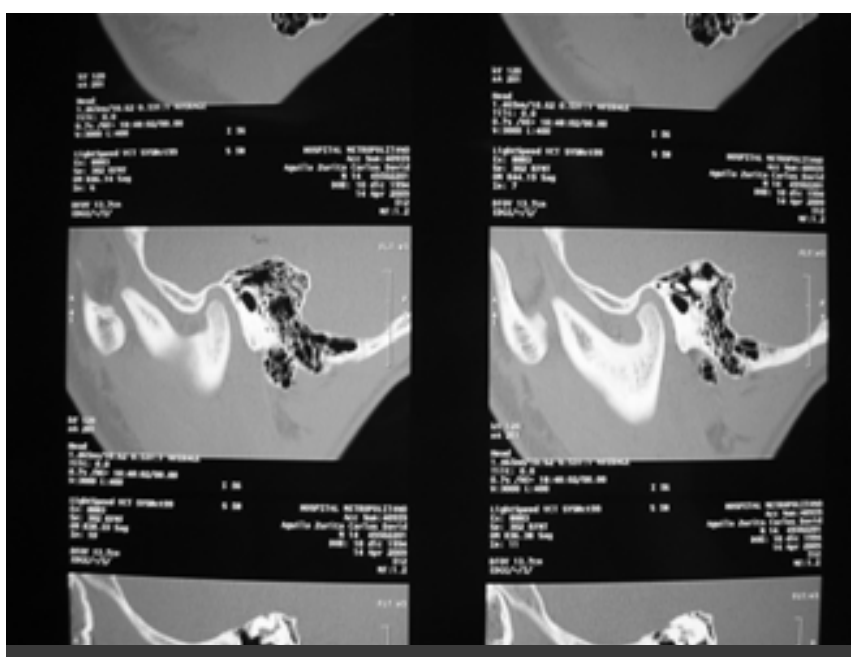

FIGURA 3. EN UN CORTE SAGITAL SE OBSERVA EL ARCO CIGOMATICO POR DELANTE DE LA APOFISIS CORONOIDES

\section{Técnica quilirúrgica}

Bajo anestesia general e intubación naso traqueal con el paciente en posición supina, se infiltró lidocaína con epinefrina al $2 \%$ a lo largo de la rama ascendente, se realizó una incisión mucoperióstica de espesor total, que se hace a lo largo de la porción inferior de la rama ascendente y se lleva hacia adelante sobre la cresta oblicua externa a la región del segundo molar. Con un elevador de periostio se disecan las fibras del músculo masetero que se insertan en la rama y ángulo mandibular, un retractor bífido se posiciona sobre el borde anterior de la rama y se retrae hacia proximal, desinsertando las fibras del 
músculo temporal, se completó la desinserción de las fibras temporales con un periostótomo para lograr una adecuada exposición del proceso coronoideo.

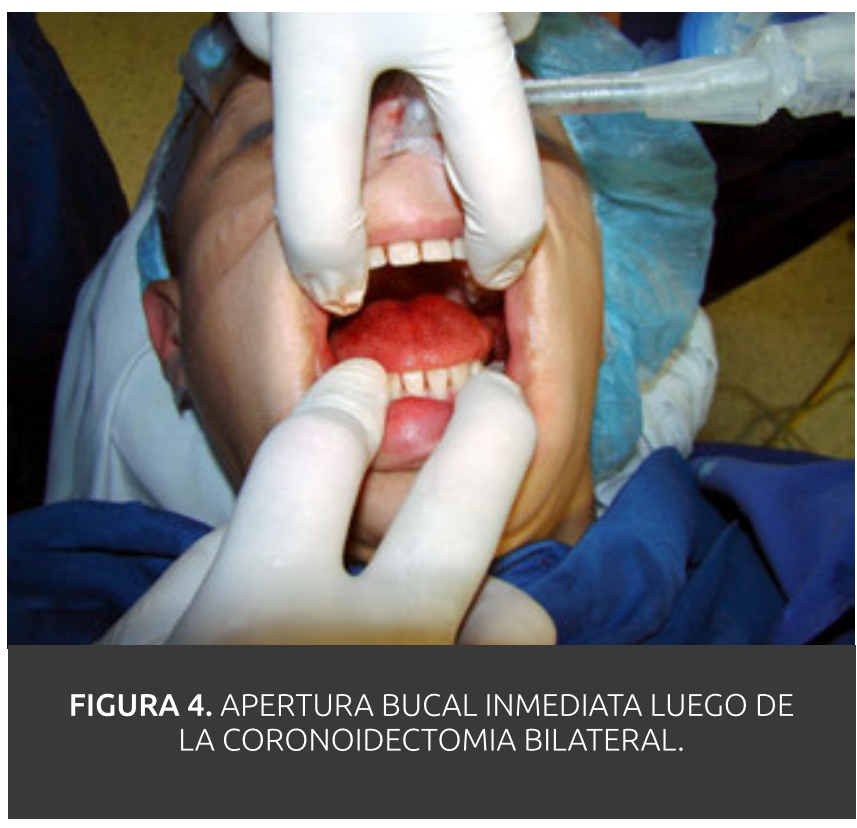

Con una cierra reciprocante se procede a la osteotomía a nivel de la base de los procesos coronoideos, inmediatamente nos permite una apertura bucal de $48 \mathrm{~mm}$ de distancia interincisal (figura 4).

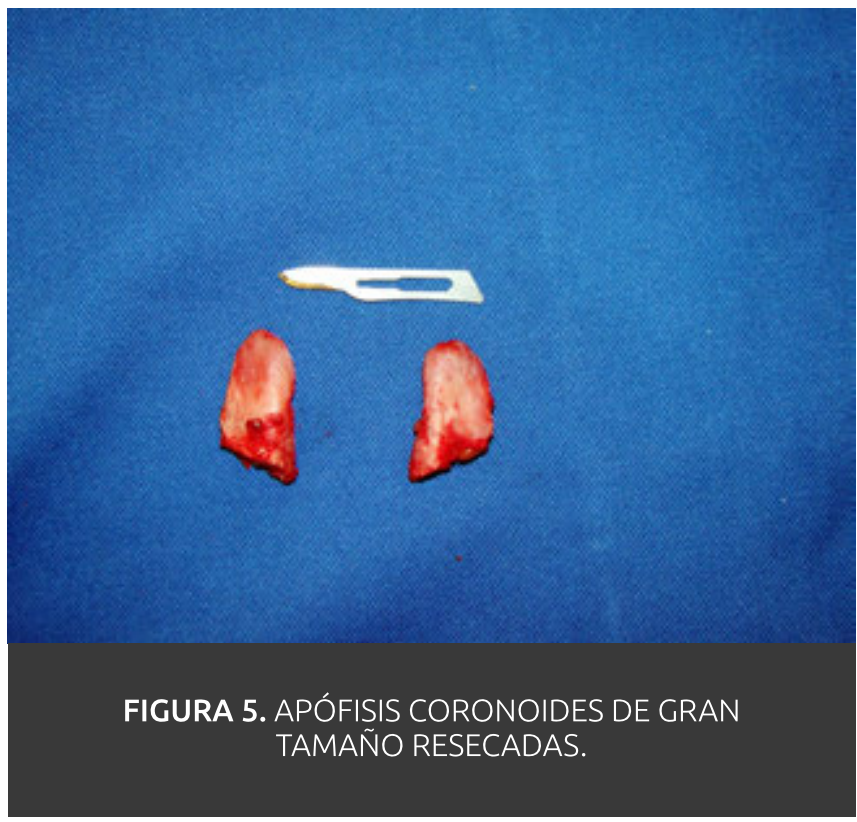

Aproximadamente $2 \mathrm{~cm}$ de cada proceso coronoideo fueron resecados (figura 5,6)
Se realizó un adecuado control de la hemostasia y posterior cierre de las incisiones mediante sutura Catgut Cromico 3-0. Posteriormente se realizó un control radiográfico donde se comprobó una adecuada osteotomía de las apófisis coronoides para no interferir en el desplazamiento mandibular.

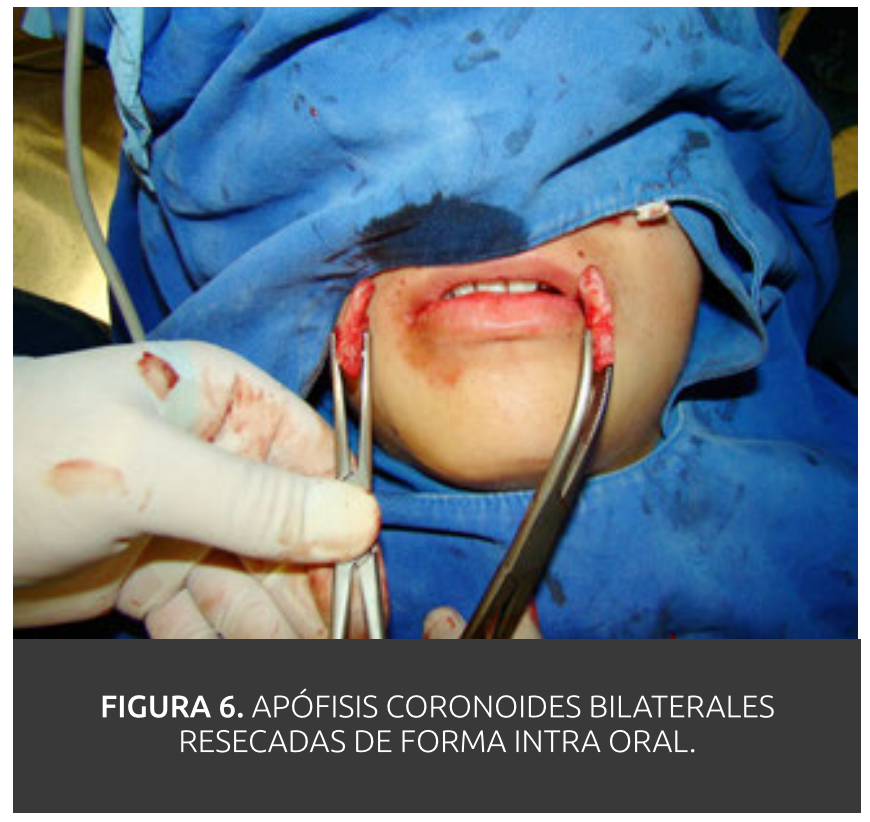

Se realizó el control post quirúrgico luego de 8 días manteniendo una adecuada y estable apertura bucal de $48 \mathrm{~mm}$. Se controló por tres meses y finalmente por un año en donde se mantuvo con una apertura de 48mm (Figura 7).

\section{Discusión}

La hiperplasia de procesos coronoides es una condición poco frecuente que puede ser unilateral o bilateral, caracterizada por la apertura bucal restringida. $\mathbf{1 , 2 , 3}$

La literatura reveló dos diferentes descripciones para los procesos coronoides hiperplásicos. La primera es la enfermedad de Jacob descrito como la formación de un osteocondroma entre el proceso coronoides y el arco cigomático que en la mayoría de los casos es unilateral, el factor causal es desconocido, pero se cree que está relacionada con la actividad perióstica anormal que forma focos del cartílago metaplásico $\mathbf{1 , 4 , 5}$. 
Según Mulder y col. Propusieron la teoría que la causa de hiperplasia de procesos coronoideos, es por mayor actividad del músculo temporal, así como la disminución de la movilidad mandibular 2,3 .

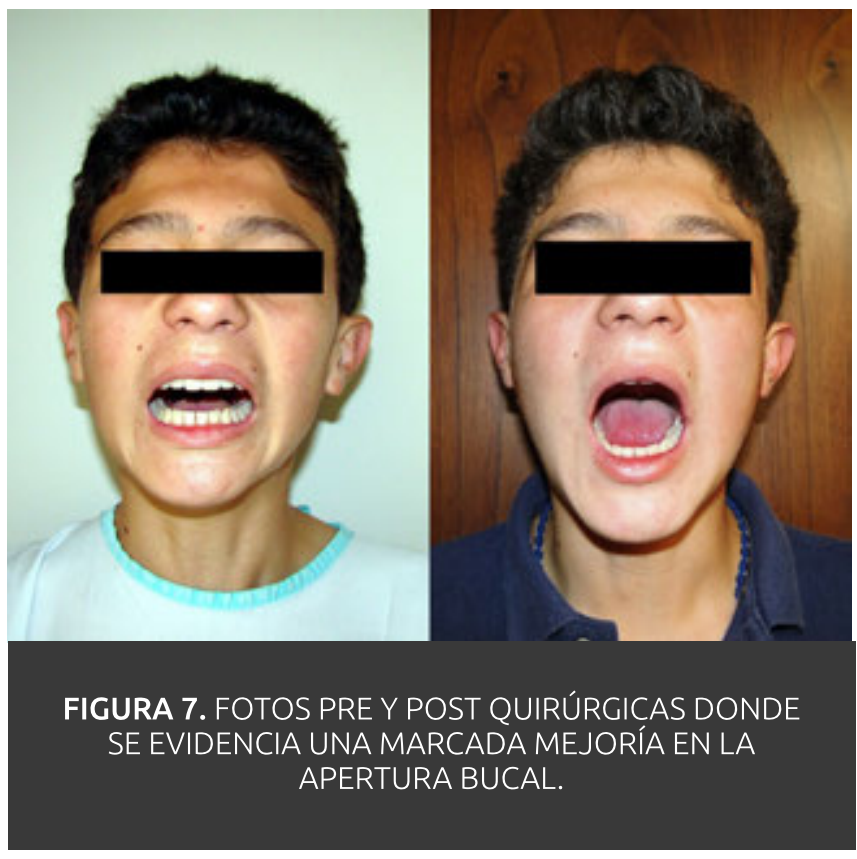

El músculo temporal es probablemente el que juegue un papel etiológico muy importante ${ }^{2,3}$. La hiperplasia de los procesos coronoideos, es el resultado de la función mecánica sobre la forma y estructura del hueso, este desarrollo es influenciado por el estrés funcional normal, (tensión y compresión). Según Sarnat y Enge encontraron que el proceso coronoideo era más grueso, más grande y largo, sobre el arco zigomático después de condilectomías realizadas en Macacus Rhesus en crecimiento y sugirieron que la hiperplasia de la coronoides fue secundaria al aumento de actividad del músculo temporal que no fue compensado por el soporte condilar $2,3,4$.

Según Washburn y col. En un estudio realizo la resección del musculo Temporal unilateral en ratas, luego de cinco meses del postoperatorio, el proceso coronoideo estuvo ausente y se consideró que se había reabsorbido como resultado de la ausencia de la tensión que ejerce el músculo temporal en la apófisis coronoides ${ }^{3}$.
En contradicción con las teorías expuestas anteriormente de hiperactividad temporal son los resultados de los estudios de electromiografía en donde el músculo temporal y los músculos maseteros, de los pacientes con hiperplasia de coronoides no se encontraron anomalías cuando los resultados se compararon con resultados de pacientes sanos 4,5. En 1999, se describieron dos casos, en los cuales la hiperplasia de coronoides se asoció con el síndrome de Moebius, este casos contradice la teoría de hiperactividad temporal $2,4,5$.

No hay pruebas concluyentes tanto para aроуаг o descartar las mencionadas teorías, y la verdadera etiología de hiperplasia de apófisis coronoides aún no está clara $\mathbf{2 , 4 , 5}$.

Según Tavassol y col. en la mayoría de los casos, el tiempo entre la aparición de los síntomas y el diagnóstico ha sido prolongado en promedio 3 años de evolución de los síntomas, la mayoría de los pacientes afectados de esta patología son de sexo masculino y las anormalidades se notaron por primera vez en el inicio de la pubertad ${ }^{\mathbf{2}, \mathbf{5}}$. La historia que manifiestan los pacientes es de una restricción progresiva de la apertura bucal, sin dolor, que comenzó varios meses antes de la consulta $2,5,6$.

Durante el examen físico, la articulación temporomandibular se encuentra asintomática, en la tomografía computarizada no se evidencian alteraciones articulares, las mismas que nos ayudan a llegar a un diagnóstico adecuado $\mathbf{2 , 3 , 4 , 5}$.

El tratamiento de la hiperplasia de procesos coronoides es siempre quirúrgico, ya sea por coronoidotomia o por coronoidectomía según Wenghoefer y col. el abordaje por vía intraoral mediante coronoidotomia de la base de la apófisis coronoides reduce el trauma quirúrgico y minimiza la exposición del hueso cuando se compara con coronoidectomias6. No obstante la mayoría de los autores en sus pacientes tratados por coronoidotomias el $95 \%$ de los casos recidivaron debido a la regeneración ósea de los 
procesos coronoides seccionados y unión fibrótica del hematoma post quirúrgico con los segmentos óseos de los procesos coronoideos ${ }^{6,7}$.

Varios abordajes extra orales se han descrito, tales como: Abordaje submandibular, preauricular, coronal o endoscópicamente, entre sus ventajas se puede mencionar menor riesgo en la formación de fibrosis y/o hematomas, y mejor exposición al resecar el proceso coronoideo y liberar el músculo temporal. $\mathbf{4 , 6 , 7}$ Los riesgo de lesión al nervio facial y una cicatriz extraoral muy antiestética son las principales desventajas de estos tipos de abordajes, a diferencia cuando se realiza un abordaje de forma intra oral $\mathbf{6 , 8 , 1 0 , 1 1}$.

Durante la coronoidectomía, las fibras musculares y tendinosas del temporal son desinsertadas completamente del proceso coronoideo permitiendo un adecuado corte de las apófisis coronoides. Entre las ventajas de realizar coronoidectomías, a diferencia de solo realizar coronoidotomías tenemos que se retira por completo la causa mecánica del bloqueo mandibular, evitando así una posible recidiva que son comunes cuando realizamos coronoidotomías ya que los segmentos óseos podrían fusionarse nuevamente. Así también las muestras óseas resecadas pueden ser enviadas para su estudio histológico y confirmar su diagnóstico $\mathbf{1 1 , 1 2 , 1 3}$. Por otro lado, la desinserción del temporal puede ser un procedimiento de difícil acceso y más traumático, pero con mejores resultados tanto estéticos, así como excelentes resultados funcionales a largo plazo 6,7,12,13.

\section{Conclusión}

Los resultados de este estudio indican que el tratamiento de la hiperplasia de procesos coronoides por vía intraoral mediante coronoidectomia bilateral, conseguimos resultados satisfactorios y estables a largo plazo, sin tendencia a recidiva en la limitación de la apertura bucal, se puede combinar con fisioterapia postoperatoria para una mayor comodidad para el paciente durante su recuperación.

\section{Referencias bibliográficas}

1. Miguel $P$, Raquel $G$, Gonzalo P. The hypothetical role of congenital hypotonia in the development of early coronoid hyperplasia. Journal of Cranio-MaxilloFacial Surgery 2012; 40: 155-158.

2. C. H. Mulder, S. I. Kalaykova, R. A. Th. Gortzak: Coronoid process hyperplasia: a systematic review of the literature from 1995. Int. J. Oral Maxillofac. Surg. 2012; 41: 1483-1489.

3. Stopa $Z$, Wanyura $H$, Kowalczyk P. Coronoid-condylar index in assessing of mandibular coronoid hyperplasia. Preliminary results. Advances in Medical Sciences Vol. 2013;58(2):429-433.

4. Carlos A, Gabriel L. Hiperplasia de procesos coronoideos mandibulares: un nuevo tratamiento posquirúrgico. Rev Esp Cir Oral Maxilofac. 2015;3 7(2):93-98.

5. F. Tavassol, S. Spalthoff, H. Essig, M. Bredt, N.C. Gellrich. Elongated coronoid process: CT-based quantitative analysis of the coronoid process and review of literature. Int. J. Oral Maxillofac. Surg. 2012; 41: 331-338.

6. Matthias $W$, Matthias $M$, Mirjam $S$. Hyperplasia of the Coronoid Process. Asian J Oral Maxillofac Surg 2006;18(1):51-58.

7. Koji S, Shigeo O, Takako A. J Oral Maxillofac Surg 2006;64:334-338.

8. J. Gagea, A. Galluccia, R. Stroumsaa. Coronoïdectomie intra-orale: note technique Transoral. Rev Stomatol Chir Maxillofac Chir Orale 2015;116:368-371. 
9. James G, Choi B, Sang $Y$, Antonio R. Bilateral Coronoid Process Hyperplasia With Pseudocartilaginous Joint Formation: Jacob Disease. J Oral Maxillofac Surg 2013;71:316-321.

10. Gerbino S, Bianchi $M$. Bernardi S. Hyperplasia of the mandibular coronoid process: long-term follow-up after coronoidotomy. Journal of CranioMaxillofacial Surgery 1997; 25, 169-173.

11. Mcloughlin C, Hopper, Bowley. Hyperplasia of the Mandibular Coronoid Process: An Analysis of 31 Cases and a Review of the Literature. J Oral Maxillofac Surg 1995; 53:250-255.

12. Masperoa C, Gianninia L, Terzib L, Sessob G. Iperplasia bilaterale del processo coronoideo in pazienti pediatrici, Bilateral hyperplasia of the coronoid process in pediatric patients. Dental CADMOS 2012;80(10):559-565.

13. Ashish C, Priya J. Coronoid hyperplasia in chronic progressive trismus. Medical Hypotheses 2011; 77:863-868.
14. G. Smyth, M. J. C. Wake. Recurrent bilateral coronoid hyperplasia: an unusual case. British Journal of Oral and Maxillofacial Surgery 1994; 32:100.104.

15. Gibbons A, Abulhoul S. Use of a Therabite appliance in the management of bilateral mandibular coronoid hyperplasia. British Journal of Oral and Maxillofacial Surgery 2007;45:505-506.

16. Annika I, Caran Z, Kyung S. Mandibular coronoid process locking: A prospective study of frequency and association with internal derangement of the temporomandibular joint. (Оral surg. oral med. oral pathol. 1987;63:275-9.

17. Shi C, Zhi j, Zhi G. Bilateral coronoid hyperplasia (Jacob disease on right and elongation on left): report of a case and literature review. Oral Med Oral Pathol Oral Radiol Endod 2009;107:e64-e67. 